\title{
INFLUÊNCIA DA TEMPERATURA DO AR DE SECAGEM NO CALOR LATENTE DE VAPORIZAÇÃO DE ÁGUA EM FEIJÃO MACASSAR (Vigna unguiculata (L.) Walp.), VARIEDADE SEMPRE-VERDE
}

\author{
WILTON P. DA SILVA ${ }^{1}$, CLEIDE M. D. P. S. E SILVA², JÜRGEN W. PRECKER ${ }^{3}$, \\ DIOGO D. P. S. E SILVA ${ }^{4}$
}

\begin{abstract}
RESUMO: Em cálculos da quantidade de energia requerida em processos de secagem artificial de um produto agrícola, é necessário o conhecimento de uma expressão para a determinação do calor latente de vaporização $(\mathrm{H})$ de água no produto. Normalmente, as expressões para $\mathrm{H}$, encontradas na literatura, são dadas pelo calor latente de vaporização (h) de água livre multiplicado por funções que dependem apenas do teor de água do produto. Isso significa que a relação $\mathrm{H} / \mathrm{h}$, para um dado produto, só depende do teor de água, o que é uma simplificação, pois se sabe que tal relação depende também da temperatura. Neste artigo, é apresentada uma expressão para o cálculo de $\mathrm{H}$ para feijão macassar, variedade sempre-verde, levando em consideração a dependência de $\mathrm{H} / \mathrm{h}$ com a temperatura. Para tal, foi desenvolvido e utilizado um programa computacional que ajusta, de forma automática, cerca de 500 funções contidas em sua biblioteca, com uma e duas variáveis independentes, a dados experimentais. O programa, que usa regressão não-linear, classifica as melhores funções ajustadas pelo critério do menor qui-quadrado reduzido. $\mathrm{O}$ conjunto de testes estatísticos realizados indica que a expressão apresentada neste artigo produz resultados mais precisos na determinação de $\mathrm{H}$ para feijão macassar que os de outras equações normalmente encontradas na literatura.
\end{abstract}

PALAVRAS-CHAVE: energia, produtos agrícolas, secagem.

\section{INFLUENCE OF THE TEMPERATURE ON THE LATENT HEAT OF VAPORIZATION OF MOISTURE FROM COWPEA (Vigna unguiculata (L.) Walp.), ALWAYS-GREEN VARIETY}

\begin{abstract}
In order to determine the energy needed to artificially dry an agricultural product the latent heat of vaporization of moisture in the product, $\mathrm{H}$, must be known. Generally, the expressions for $H$ reported in the literature are of the form $H=h(T) f(M)$, where $h(T)$ is the latent heat of vaporization of free water, and $\mathrm{f}(\mathrm{M})$ is a function of the equilibrium moisture content, $\mathrm{M}$, which is a simplification. In this article, a more general expression for the latent heat of vaporization, namely $\mathrm{H}=\mathrm{g}(\mathrm{M}, \mathrm{T})$, is used to determine $\mathrm{H}$ for cowpea, always-green variety. For this purpose, a computer program was developed which automatically fits about 500 functions, with one or two independent variables, imbedded in its library to experimental data. The program uses nonlinear regression, and classifies the best functions according to the least reduced chi-squared. A set of executed statistical tests shows that the generalized expression for $\mathrm{H}$ used in this work produces better results of $\mathrm{H}$ for cowpea than other equations found in literature.
\end{abstract}

KEYWORDS: energy, agricultural products, drying.

\footnotetext{
${ }^{1}$ Prof. Adjunto, DF/CCT/UFCG, Campina Grande - PB, Fone: (0XX83) 3333.2962, wiltonps@uol.com.br

${ }^{2}$ Prof. Adjunto, DF/CCT/UFCG, Campina Grande - PB, cleidedps@uol.com.br

${ }^{3}$ Prof. Associado, DF/CCT/UFCG, Campina Grande - PB, jurgenp@uol.com.br

${ }^{4}$ Doutorando, UNICAMP, Instituto de Matemática, Estatística e Computação Científica (IMECC), Campinas - SP, diogodme@gmail.com

Recebido pelo Conselho Editorial em: $1^{0}$-2-2007

Aprovado pelo Conselho Editorial em: 25-2-2008
} 


\section{INTRODUÇÃO}

No Brasil, em particular na região Nordeste, percentual significativo da população consome feijão macassar regularmente, tanto in natura quanto industrializado. No segundo caso, o produto é previamente desidratado e reidratado no momento do preparo. Na operação de secagem artificial do produto, um fator que merece especial atenção é a quantidade de energia consumida no processo, devido ao seu custo.

Em estudos referentes à secagem artificial de produto agrícola, a determinação da quantidade de energia requerida no processo depende, dentre vários fatores, do calor latente de vaporização $(\mathrm{H})$ de água no produto. Como $\mathrm{H}$, em geral, é significativamente diferente do calor latente de vaporização $(\mathrm{h})$ de água livre e dependente do produto investigado, muitas pesquisas têm sido desenvolvidas com o objetivo de determinar expressões para o cálculo de $\mathrm{H}$ para vários produtos.

BROOK \& FOSTER (1981), citados por BROOKER et al. (1992), determinaram expressões para o cálculo de $\mathrm{H}$ para vários produtos, a partir de dados experimentais, com a pressuposição de que a relação $\mathrm{H} / \mathrm{h}$ para um dado produto pudesse ser expressa por uma função que dependia unicamente do teor de água do produto. Tais expressões são usadas em trabalhos sobre simulações de secagem de produtos agrícolas, como, por exemplo, em MATA et al. (1999) e em CAVALCANTI MATA \& DUARTE (2003).

CORRÊA et al. (1998) modificaram a função que relaciona $\mathrm{H} / \mathrm{h}$ com o teor de água, proposta por BROOK \& FOSTER (1981), e ajustaram a função modificada a dados obtidos para milho pipoca. KALEEMULLAH \& KAILAPPAN (2005) determinaram uma expressão para o cálculo de $\mathrm{H}$ para pimenta-vermelha a partir de dados isotérmicos obtidos para esse produto. Em todos esses trabalhos, a dependência da relação $\mathrm{H} / \mathrm{h}$ com a temperatura do produto não foi considerada.

Neste trabalho, teve-se como objetivo investigar a dependência da relação $\mathrm{H} / \mathrm{h}$ com a temperatura de um produto e verificar se essa dependência é significativa na determinação de uma expressão para H. Outro objetivo foi buscar formas de incluir tal dependência na determinação de uma expressão empírica para o cálculo de $\mathrm{H}$ para feijão macassar, variedade sempre-verde, e comparar a expressão obtida com outras existentes na literatura.

\section{MATERIAL E MÉTODOS}

Para obter uma expressão para o cálculo do calor latente de vaporização $(\mathrm{H})$ da água em um produto, normalmente, a equação de Clausius-Clapeyron (ver, por exemplo, SMITH et al., 2000) é utilizada, com a suposição de que a equação de estado dos gases ideais possa ser aplicada ao vapor, desconsiderando-se o volume de água na fase líquida. O procedimento comum na solução da equação de Clausius-Clapeyron é fazer a sua integração, considerando $\mathrm{H}$ constante entre dois estados estabelecidos. Para a água livre, o calor latente de vaporização (h) é obtido com o mesmo raciocínio anterior, substituindo-se a pressão de vapor $\left(\mathrm{P}_{\mathrm{v}}\right)$ pela pressão de vapor saturado $\left(\mathrm{P}_{\mathrm{vs}}\right)$. Dessa forma, relembrando que $\mathrm{P}_{\mathrm{v}}=\Phi \mathrm{P}_{\mathrm{vs}}$, em que $\Phi$ é a umidade relativa do ar, e comparando a expressão obtida para $\mathrm{H}$ com aquela obtida para $\mathrm{h}$, ambas na mesma faixa de temperatura, tem-se a eq.(1) (BROOKER et al., 1992):

$$
\frac{\mathrm{H}}{\mathrm{h}}=\frac{\ln \left(\Phi_{1} \mathrm{P}_{\mathrm{vs} 1}\right)-\ln \left(\Phi_{2} \mathrm{P}_{\mathrm{vs} 2}\right)}{\ln \mathrm{P}_{\mathrm{vs} 1}-\ln \mathrm{P}_{\mathrm{vs} 2}}
$$

Uma vez determinados $\Phi$ e $\mathrm{P}_{\mathrm{vs}}$ para os estados 1 e 2 , a relação $\mathrm{H} / \mathrm{h}$, definida pela eq.(1), torna-se conhecida. A umidade relativa do ar $(\Phi)$ pode ser obtida por quaisquer das equações disponíveis na literatura, para cálculo de teor de água de equilíbrio, tais como: Chung, Copace, Henderson, Halsey, Chung-Pfost, Sabbah, Sigma-Copace e Cavalcanti Mata. Entretanto, foi usada a equação obtida por SILVA et al. (2005), devido ao fato de essa equação apresentar melhores indicadores de ajuste para dados de feijão macassar, variedade sempre-verde. Tais dados foram disponibilizados por OLIVEIRA et al. (2004) e, para o referido produto, utilizou-se da eq.(2): 


$$
\mathrm{M}=\frac{\mathrm{e}^{-0,007726 \mathrm{~T}}}{\ln (1,0925-0,000886 \Phi+1 / \Phi)} \times 0,01
$$

em que,

$\mathrm{T}$ - temperatura, ${ }^{\circ} \mathrm{C}$;

$\Phi$ - umidade relativa do ar, \%, e

$\mathrm{M}$ - teor de água de equilíbrio (decimal, em base seca).

Dados M e T, a umidade relativa do ar $(\Phi)$ pode ser determinada a partir da eq.(2), que pode ser transformada em uma equação do segundo grau em $\Phi$. Entretanto, a forma mais cômoda para a determinação de $\Phi$ é a utilização de um programa computacional com opção para cálculo de raízes de equações transcendentais, como, por exemplo, o LAB Fit ${ }^{\circledR}$ (SILVA et al., 2004).

A pressão de vapor saturado pode ser calculada a partir, por exemplo, dos dados experimentais disponíveis em BROOKER et al. (1992), ou ainda em SMITH et al. (2000). Dentre as equações com três parâmetros, pode-se usar a equação de Antoine, disponível em COSTA et al. (1999), por meio do programa computacional ELV. Entretanto, os cálculos de $\mathrm{P}_{\mathrm{vs}}$ foram efetuados utilizando a equação de Riedel (ATHIÉ et al., 1998; SHORE et al., 2002) devido ao fato de essa equação apresentar melhores indicadores de ajuste aos dados experimentais. Neste trabalho, os parâmetros da equação de Riedel foram obtidos por meio de regressão não-linear, utilizando os dados disponibilizados por BROOKER et al. (1992). Assim, tem-se a eq.(3):

$$
\mathrm{P}_{\text {vs }}=\mathrm{e}^{[49,20-6643 /(\mathrm{T}+273,15)-4,522 \ln (\mathrm{T}+273,15)]}
$$

em que, $\mathrm{P}_{\mathrm{vs}}$ é obtido em $\mathrm{kPa}$ quando $\mathrm{T}$ é dado em ${ }^{\circ} \mathrm{C}$.

Com as eqs.(2) e (3), podem-se determinar, respectivamente, $\Phi$ e $\mathrm{P}_{\mathrm{vs}}$ para valores estipulados de $\mathrm{M}$ e T, o que possibilita determinar a relação H/h por meio da eq.(1). Por outro lado, a eq.(4) para o calor latente de vaporização de água livre é dada por BROOKER et al. (1992):

$$
\mathrm{h}(\mathrm{T})=2.503-2,386 \mathrm{~T}
$$

em que, h é obtido em $\mathrm{kJ} \mathrm{kg}^{-1}$ para a temperatura em ${ }^{\circ} \mathrm{C}$.

Em trabalhos sobre a determinação de calor latente de vaporização de água em um dado produto, é comum admitir que a relação $\mathrm{H} / \mathrm{h}$, num dado intervalo de temperatura, só dependa do teor de água de tal produto. BROOK \& FOSTER (1981), citados por BROOKER et al. (1992), propuseram a eq.(5):

$$
\frac{\mathrm{H}}{\mathrm{h}}=1+\mathrm{Ae}^{\mathrm{BM}}
$$

em que os parâmetros $\mathrm{A}$ e $\mathrm{B}$ são determinados por ajuste da função aos dados obtidos para o produto em estudo.

CORRÊA et al. (1998) propuseram uma modificação na função [eq.(6)], definida pela eq.(5):

$$
\frac{\mathrm{H}}{\mathrm{h}}=1+\mathrm{Ae}^{\mathrm{BM}}
$$

Levando em conta a eq.(4), uma vez conhecidos os parâmetros das eqs.(5) e (6), essas podem ser reescritas de forma a possibilitar o cálculo do calor latente de vaporização de água para um dado produto [eqs.(7) e (8)]: 


$$
H=(2.503-2,386 \mathrm{~T})\left(1+A \mathrm{e}^{\mathrm{BM}}\right)
$$

$\mathrm{e}$

$$
\mathrm{H}=(2.503-2,386 \mathrm{~T})\left(1+\mathrm{Ae}^{\mathrm{BM}}{ }^{\mathrm{C}}\right)
$$

em que, A, B e C possuem valores específicos para cada produto.

Obviamente, nas eqs.(7) e (8), é suposto que o calor latente de vaporização de água no produto seja dado por uma função expressa por duas outras, com separação de variáveis, na forma da eq.(9):

$$
\mathrm{H}=\mathrm{h}(\mathrm{T}) \mathrm{f}(\mathrm{M})
$$

Neste trabalho, foi investigado se uma função mais geral, sem a prévia suposição de separação de variáveis, dada na forma expressa pela eq.(10), descreve melhor o calor latente de vaporização de água $(\mathrm{H})$, em um produto.

$$
\mathrm{H}=\mathrm{g}(\mathrm{M}, \mathrm{T})
$$

O procedimento utilizado na investigação foi um delineamento casualizado, em esquema fatorial $5 \times 4$, sendo cinco níveis para o teor de água, $M(0,10 ; 0,15 ; 0,20 ; 0,25$ e $0,30 \mathrm{bs})$, e quatro níveis para a temperatura, $\mathrm{T}\left(20 ; 30 ; 40\right.$ e $\left.50{ }^{\circ} \mathrm{C}\right)$.

$\mathrm{O}$ cálculo de $\mathrm{H} / \mathrm{h}$, por meio da eq.(1), para um dado teor de água (M), numa temperatura específica (T), pressupõe o conhecimento da umidade relativa do ar $(\Phi)$ em dois estados, denominados de 1 e 2 . Assim, a umidade relativa do ar foi calculada por meio da eq.(2), num estado 1 , com $\mathrm{T}_{1}=\mathrm{T}+2{ }^{\circ} \mathrm{C}$, e num estado 2 , com $\mathrm{T}_{2}=\mathrm{T}-2{ }^{\circ} \mathrm{C}$. A rigor, os dois estados em torno de $\mathrm{T}$ poderiam ser caracterizados por outros valores, como, por exemplo, 0,5 ou $1{ }^{\circ} \mathrm{C}$, ao invés de $2{ }^{\circ} \mathrm{C}$. Entretanto, a faixa escolhida, definida por $2{ }^{\circ} \mathrm{C}$, produz os mesmos resultados que as faixas definidas por esses outros valores e, por isso, tal faixa foi utilizada nos cálculos efetuados.

A pressão de vapor saturado foi calculada por meio da eq.(3), e isso possibilitou calcular a relação $\mathrm{H} / \mathrm{h}$, utilizando a eq.(1) para a matriz estipulada. Com isso, pôde-se determinar $\mathrm{H}$ para cada $\mathrm{M}$ e T, uma vez que $\mathrm{h}(\mathrm{T})$ pode ser calculado pela eq.(4). Assim, por meio de ajuste de curvas, puderam-se determinar os parâmetros $\mathrm{A}$ e B da eq.(7) e A, B e C da eq.(8).

A análise dos resultados para os pontos $(\mathrm{M}, \mathrm{T}, \mathrm{H} / \mathrm{h})$ deve indicar se a consideração de valor constante para $\mathrm{H} / \mathrm{h}$ em relação à temperatura é adequada ou não. Se não for, pode-se tentar a determinação de outra expressão para $\mathrm{H}$ em função de $\mathrm{M}$ e T. Para tal, utilizou-se da opção Finder, que é uma ferramenta do programa LAB Fit ${ }^{\circ}$, que ajusta, de forma automática, cerca de 500 funções compactas de uma e duas variáveis independentes contidas em sua biblioteca aos dados experimentais. Essa ferramenta foi desenvolvida pelos dois primeiros autores deste trabalho e incorporada ao LAB Fit ${ }^{\circledR}$ a partir da versão 7.2.18. O principal objetivo do Finder é determinar equações empíricas visando à descrição de dados experimentais. Vale salientar que o Finder classifica as melhores funções ajustadas pelo critério de menor qui-quadrado reduzido.

Uma vez determinada uma expressão para $\mathrm{H}$, por meio do Finder, realizou-se comparação entre essa expressão e aquelas obtidas por ajustes das Eqs.(7) e (8). Tal comparação foi realizada com base nos seguintes testes estatísticos: coeficiente de determinação, qui-quadrado reduzido e teste $\mathrm{t}$ de Student.

\section{RESULTADOS E DISCUSSÃO}

Para os valores estipulados para a temperatura e para o teor de água de feijão macassar, pôdese montar a Tabela 1, que indica a umidade relativa do ar ( $\Phi)$ calculada por meio da eq.(2), correspondente a cada par T e M. 
TABELA 1. Umidade relativa do ar ( $\Phi)$ obtida pela eq.(2) para feijão macassar. Relative umidity of air ( $\Phi)$ for cowpea obtained by eq.(2).

\begin{tabular}{ccccccccc}
\hline & \multicolumn{8}{c}{$\mathrm{T}\left({ }^{\circ} \mathrm{C}\right)$} \\
\cline { 2 - 9 } M (bs) & \multicolumn{2}{c}{20} & \multicolumn{2}{c}{30} & & 40 & \multicolumn{2}{c}{50} \\
\cline { 2 - 9 } & Est. 2 & Est. 1 & Est. 2 & Est. 1 & Est. 2 & Est. 1 & Est. 2 & Est. 1 \\
\cline { 2 - 9 } & 18 & 22 & 28 & 32 & 38 & 42 & 48 & 52 \\
\hline 0,10 & 0,3450 & 0,3621 & 0,3880 & 0,4055 & 0,4317 & 0,4491 & 0,4750 & 0,4921 \\
0,15 & 0,5683 & 0,5840 & 0,6071 & 0,6221 & 0,6439 & 0,6581 & 0,6787 & 0,6920 \\
0,20 & 0,7025 & 0,7153 & 0,7338 & 0,7457 & 0,7630 & 0,7741 & 0,7903 & 0,8007 \\
0,25 & 0,7873 & 0,7978 & 0,8130 & 0,8228 & 0,8369 & 0,8460 & 0,8592 & 0,8676 \\
0,30 & 0,8451 & 0,8540 & 0,8668 & 0,8750 & 0,8870 & 0,8946 & 0,9057 & 0,9128 \\
\hline
\end{tabular}

Por outro lado, usando a eq.(3), pôde-se determinar a pressão de vapor saturado para água livre, nas vizinhanças de cada temperatura estipulada: $\mathrm{P}_{\mathrm{vs} 1}\left(\mathrm{~T}_{1}=\mathrm{T}+2{ }^{\circ} \mathrm{C}\right)$ e $\mathrm{P}_{\mathrm{vs} 2}\left(\mathrm{~T}_{2}=\mathrm{T}-2{ }^{\circ} \mathrm{C}\right)$, o que resulta na Tabela 2 .

TABELA 2. Pressão de vapor saturado obtida pela eq.(3). Saturated vapour pressure obtained by eq.(3).

\begin{tabular}{ccccc}
\hline $\mathrm{T}\left({ }^{\circ} \mathrm{C}\right)$ & 20 & 30 & 40 & 50 \\
\hline $\mathrm{P}_{\mathrm{vs} 1}(\mathrm{kPa})$ & 2,6485 & 4,7631 & 8,2142 & 13,638 \\
$\mathrm{P}_{\mathrm{vs} 2}(\mathrm{kPa})$ & 2,0678 & 3,7864 & 6,6369 & 11,183 \\
\hline
\end{tabular}

Com os resultados obtidos nas Tabelas 1 e 2, a eq.(1) pode ser usada para a determinação da relação $\mathrm{H} / \mathrm{h}$ para os valores da temperatura e do teor de água previamente estipulados, o que resultou na Tabela 3.

TABELA 3. Relação H/h calculada pela eq.(1) para feijão macassar. Relation H/h for cowpea calculated by eq.(1).

\begin{tabular}{ccccc}
\hline \multirow{2}{*}{$\mathrm{M}(\mathrm{bs})$} & \multicolumn{5}{c}{$\mathrm{T}\left({ }^{\circ} \mathrm{C}\right)$} \\
\cline { 2 - 5 } & 20 & 30 & 40 & 50 \\
\hline 0,10 & 1,1955 & 1,1922 & 1,1853 & 1,1782 \\
0,15 & 1,1101 & 1,1064 & 1,1023 & 1,0979 \\
0,20 & 1,0730 & 1,0701 & 1,0677 & 1,0659 \\
0,25 & 1,0535 & 1,0522 & 1,0507 & 1,0490 \\
0,30 & 1,0423 & 1,0410 & 1,0400 & 1,0393 \\
\hline
\end{tabular}

Observando-se a Tabela 3, verifica-se que existe suave dependência entre $\mathrm{H} / \mathrm{h}$ e a temperatura, para um dado teor de água. Para o teor de água igual a 0,10, a discrepância percentual de $\mathrm{H} / \mathrm{h}$ entre 20 e $50{ }^{\circ} \mathrm{C}$ é de, aproximadamente, $1,45 \%$. Torna-se evidente, então, a introdução de erro sistemático ao descrever tal relação pelas eqs.(5) ou (6). Isso pode ser visualizado pelo ajuste da eq.(6) aos dados da Tabela 3, que gera o gráfico observado na Figura 1, em que todos os pontos referentes às temperaturas de 40 e $50{ }^{\circ} \mathrm{C}$ se situam abaixo da superfície de resposta.

Por outro lado, o calor latente de vaporização para água livre pode ser obtido pela eq.(4), para as temperaturas estipuladas, o que é dado na Tabela 4.

Uma vez obtidos os valores de $\mathrm{H} / \mathrm{h}$, dados na Tabela 3 , e de $\mathrm{h}$, dados na Tabela 4 , o calor latente de vaporização $(\mathrm{H})$ de água em feijão macassar pode ser obtido pelo produto das duas grandezas, o que gera a Tabela 5 . 


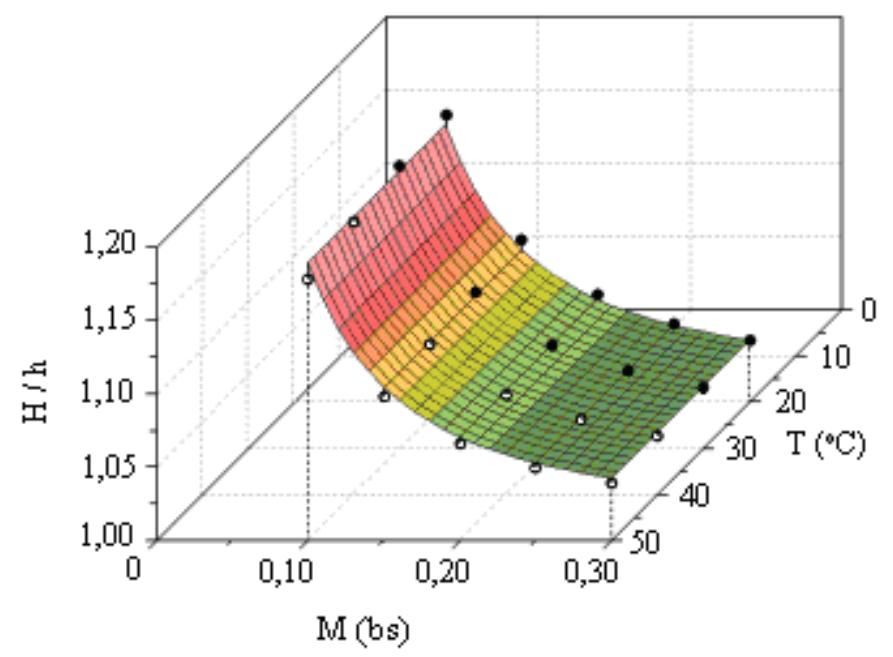

FIGURA 1. Eq.(6) ajustada aos dados da Tabela 3. Círculos cheios representam dados experimentais acima ou sobre a superfície de resposta, e círculos vazios, abaixo dessa superficie. Eq.(6) fitted to the data of Table 3. Full circles represent experimental data lying on or above the surface, empty circles represent data below.

TABELA 4. Calor latente de vaporização (h) para água livre obtido pela eq.(4). Latent heat of vaporization (h) of free water obtained by eq.(4).

\begin{tabular}{ccccc}
\hline $\mathrm{T}\left({ }^{\circ} \mathrm{C}\right)$ & 20 & 30 & 40 & 50 \\
$\mathrm{~h}\left(\mathrm{~kJ} \mathrm{~kg}^{-1}\right)$ & 2.455 & 2.431 & 2.408 & 2.384 \\
\hline
\end{tabular}

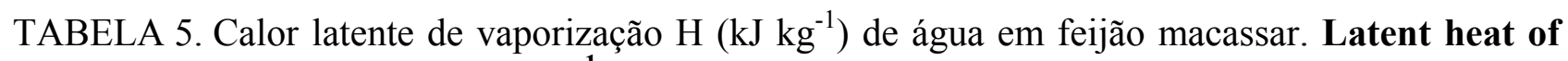
vaporization $H\left(\mathrm{~kJ} \mathrm{~kg}^{-1}\right)$ of water in cowpea.

\begin{tabular}{ccccc}
\hline \multirow{2}{*}{$\mathrm{M}(\mathrm{bs})$} & \multicolumn{3}{c}{$\mathrm{T}\left({ }^{\circ} \mathrm{C}\right)$} \\
\cline { 2 - 5 } & 20 & 30 & 40 & 50 \\
\hline 0,10 & 2.935 & 2.898 & 2.854 & 2.809 \\
0,15 & 2.725 & 2.690 & 2.654 & 2.617 \\
0,20 & 2.634 & 2.601 & 2.571 & 2.541 \\
0,25 & 2.586 & 2.558 & 2.530 & 2.501 \\
0,30 & 2.559 & 2.531 & 2.504 & 2.478 \\
\hline
\end{tabular}

Um ajuste da função dada pela eq.(7) aos dados da Tabela 5 produziu a eq.(11):

$$
\mathrm{H}=(2.503-2,386 \mathrm{~T})\left(1+0,4501 \mathrm{e}^{-9,060 \mathrm{M}}\right)
$$

com $\mathrm{H}$ em kJ kg${ }^{-1}$ e $\mathrm{T} \mathrm{em}{ }^{\circ} \mathrm{C}$.

Vale salientar que a eq.(11) envolve quatro parâmetros, embora só dois tenham sido determinados por ajuste de curvas.

Com relação ao ajuste, são apresentados os seguintes resultados para os testes estatísticos realizados. Quanto ao qui-quadrado reduzido, cuja raiz quadrada, para o tipo de dados analisados, é igual ao desvio-padrão associado ao ajuste, obteve-se $\chi_{\text {red }}^{2}=520,529$.

Com relação ao coeficiente de determinação, que indica a proporção ou o percentual de variação explicada pela regressão, obteve-se $\mathrm{R}^{2}=0,9747916$. Para o teste $\mathrm{t}$, que dá a probabilidade de um parâmetro ser zero, mesmo tendo o valor obtido, obteve-se $\mathrm{P}(\mathrm{t})=0$ para os dois parâmetros determinados, o que indica que eles são significativos. Detalhes sobre esses testes estatísticos podem ser obtidos em BEVINGTON \& ROBINSON (1992), BUSSAB \& MORETTIN (1995), 
TAYLOR (1997), SILVA \& SILVA (1998) e em BARROS NETO et al. (2003). Um gráfico para a função $\mathrm{H}(\mathrm{M}, \mathrm{T})$ obtida pela eq.(11) é apresentado na Figura 2.

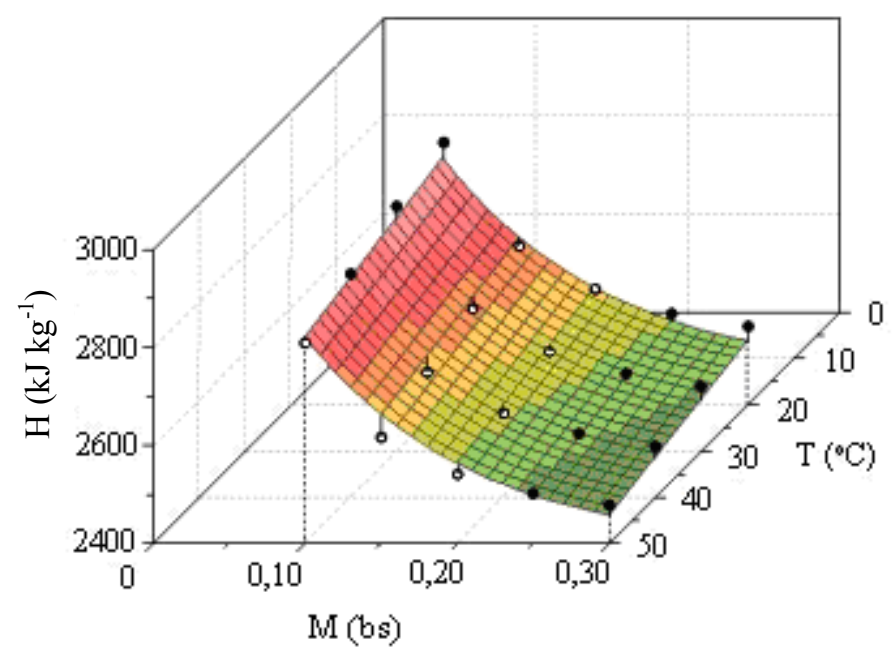

FIGURA 2. Gráfico de H(M,T) obtido pelo modelo proposto por meio da eq.(11). Plot of H(M,T) obtained by the proposed model by eq.(11).

Para a eq.(8), ajustada aos dados da Tabela 5, obteve-se a eq.(12):

$$
\mathrm{H}=(2.503-2,386 \mathrm{~T})\left(1+1,9774 \times 10^{-6} \mathrm{e}^{8,4791 \mathrm{M}^{-0,13091}}\right)
$$

com $\mathrm{Hem} \mathrm{kJ} \mathrm{kg}{ }^{-1}$ e $\mathrm{T} \mathrm{em}{ }^{\circ} \mathrm{C}$.

A eq.(12) envolve cinco parâmetros, dos quais três foram determinados por ajuste de curvas. Com relação aos testes estatísticos referentes ao ajuste, foi obtido $\mathrm{R}^{2}=0,9954851$ e $\chi_{\text {red }}^{2}=98,602$. $\mathrm{O}$ teste $\mathrm{t}$ de Student para o primeiro parâmetro determinado indica que existe probabilidade de $92,3 \%$ de ele ser zero, mesmo tendo o valor $1,9774 \times 10^{-6}$. Já o segundo parâmetro tem probabilidade de ser zero de $12,4 \%$, e o terceiro de $32,4 \%$. O gráfico $\mathrm{H}(\mathrm{M}, \mathrm{T})$, referente à eq.(12), é mostrado na Figura 3.

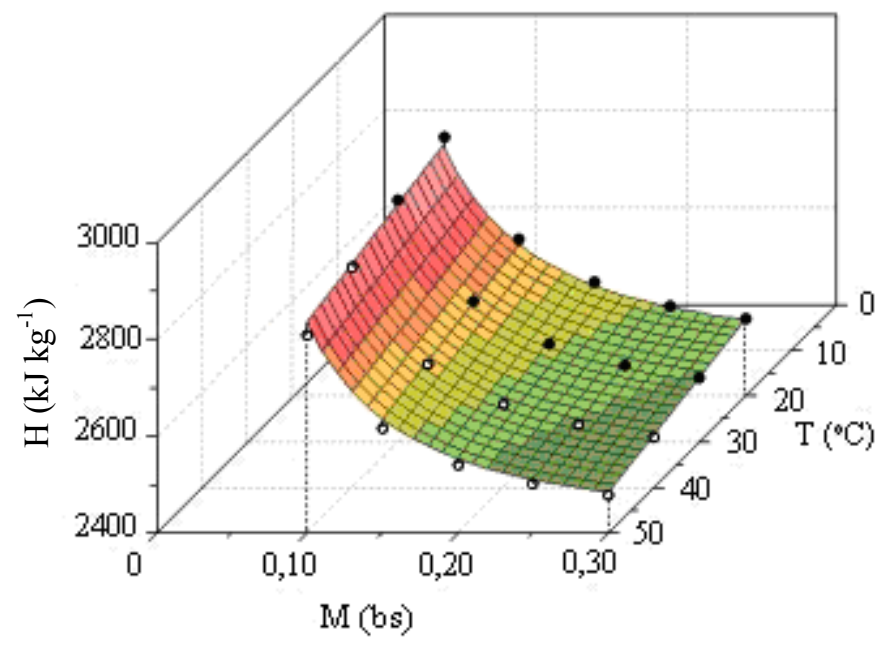

FIGURA 3. Gráfico de H(M,T) obtido pelo modelo proposto por meio da eq.(12). Plot of H(M,T) obtained by the proposed model by eq.(12).

Dois dos indicadores da qualidade dos ajustes realizados $\left(\chi_{\text {red }}^{2}\right.$ e $\left.\mathrm{R}^{2}\right)$ possibilitariam afirmar que a eq.(12) é mais adequada para descrever os dados obtidos na Tabela 5 do que a eq.(11). CORREA et al. (1998) já haviam chegado a conclusão semelhante ao estudar sementes de milho- 
pipoca. Entretanto, no caso específico de feijão macassar, há que ser observado que os três parâmetros da eq.(12), determinados por ajuste de curvas, têm considerável probabilidade de ser zero, mesmo tendo os valores obtidos. Isso significa que o modelo dado pela eq.(12) deve ser rejeitado como expressão para o cálculo do calor latente de vaporização de água em feijão macassar.

Como a inspeção da Tabela 3 indica suave dependência da relação H/h com a temperatura, funções do tipo indicado pela eq.(9), dadas por separação de variáveis, podem não ser as mais adequadas para expressar o calor latente de vaporização de água em um produto. Assim, utilizando o Finder do LAB Fit ${ }^{\circledR}$ para os dados da Tabela 5, o programa computacional relacionou as melhores funções dentre as existentes em sua biblioteca. A que melhor representa os dados da Tabela 5 é a função identificada pelo número de ordem igual a 408. Feito o ajuste dessa função aos dados, obteve-se a eq.(13):

$$
\mathrm{H}=494,462 \mathrm{e}^{[(0,0765 / \mathrm{M})-0,004949 \mathrm{~T}]}+1978,4
$$

com $\mathrm{H} \mathrm{em} \mathrm{kJ} \mathrm{kg}{ }^{-1}$ e $\mathrm{T}$ em ${ }^{\circ} \mathrm{C}$.

A eq.(13) envolve quatro parâmetros, todos determinados por ajuste de curvas. Com relação aos testes estatísticos referentes à regressão não-linear dessa função, foram obtidos $\mathrm{R}^{2}=0,9996 \mathrm{e}$ $\chi_{\text {red }}^{2}=8,201$. Nesse ajuste, todos os parâmetros determinados são significativos, pois, para todos eles, o teste $\mathrm{t}$ de Student aponta para $\mathrm{P}(\mathrm{t})=0$. Os indicadores da qualidade desse último ajuste são bem melhores que aqueles referentes às eqs.(11) e (12) e, por isso, a eq.(13) pode ser considerada como uma opção para a determinação de calor latente de vaporização de água em feijão macassar. O gráfico da função H(M, T), referente à eq.(13), é apresentado na Figura 4.

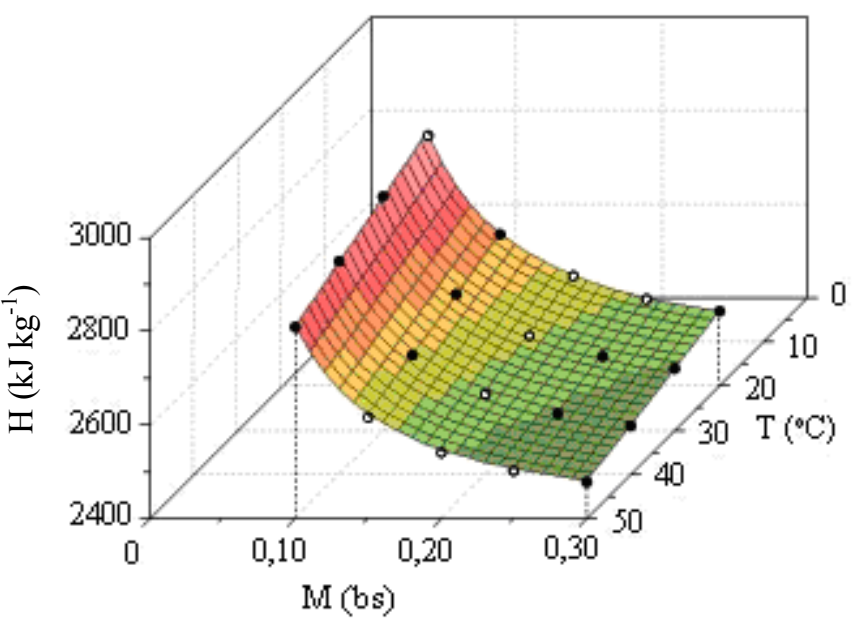

FIGURA 4. Gráfico de H(M,T) obtido pelo modelo proposto por meio da eq.(13). Plot of H(M,T) obtained by the proposed model by eq.(13).

Para que se tenha uma idéia da precisão de um resultado obtido pela eq.(13), em comparação com o valor correspondente na Tabela 5, foi feito o cálculo de $\mathrm{H}$ para $\mathrm{M}=0,10$ e $\mathrm{T}=20{ }^{\circ} \mathrm{C}$. Para tais valores de $\mathrm{M}$ e $\mathrm{T}$, têm-se $\mathrm{H}=2.941 \mathrm{~kJ} \mathrm{~kg}^{-1}$ [eq.(13)] e $\mathrm{H}=2.935 \mathrm{~kJ} \mathrm{~kg}^{-1}$ (Tabela 5), o que significa que os dois valores têm discrepância percentual de apenas $0,2 \%$.

\section{CONCLUSÕES}

Como a relação $\mathrm{H} / \mathrm{h}$ diminui ligeiramente com o aumento da temperatura, para um dado teor de água, funções do tipo dado pela eq.(10) são mais indicadas que aquelas dadas pela eq.(9) para a determinação, por ajuste de curvas, de uma expressão para o calor latente de vaporização de água do produto. 
Os indicadores da qualidade do ajuste da eq.(13) aos dados da Tabela 5 sugerem que tal equação se constitui em uma opção adequada para a determinação do calor latente de vaporização do produto analisado.

Os resultados obtidos para o produto estudado sugerem que a metodologia proposta neste artigo pode constituir-se em alternativa adequada para a determinação de expressões para o calor latente de vaporização de água em outros produtos.

\section{REFERÊNCIAS}

ATHIÉ, I.; CASTRO, M.F.P.M.; GOMES, R.A.R.; VALENTINI, S.R.T. Conservação de grãos. Campinas: Fundação Cargill, 1998. 236 p.

BARROS NETO, B.; SCARMÍNIO, I. S.; BRUNS, R. E. Como fazer experimentos. Campinas: UNICAMP, 2003. $401 \mathrm{p}$.

BEVINGTON, P.R.; ROBINSON, D.K. Data reduction and error analysis for the physical sciences. $2^{\text {nd }}$ ed. Boston: WCB/McGraw-Hill, 1992.328 p.

BROOK, R.C.; FOSTER, G.H. Drying, cleaning and conditioning. In: CRC Handbook of transportation, marketing and agriculture. Boca Raton: CRC Press, 1981. v.2.

BROOKER, D.B.; BAKKER-ARKEMA, F.W.; HALL, C.W. Drying and storage of grains and oilseeds. Westport: The AVI Publishing Company, 1992. 450 p.

BUSSAB, W.O.; MORETTIN, P. A estatística básica. São Paulo: Atual Editora, 1995. 322 p.

CAVALCANTI MATA, M.E.R.M.; DUARTE, M.E.M. Drying simulation theory of the cowpea considering the grains shrinkage. Revista Brasileira de Produtos Agroindustriais, Campina Grande, v.5, n.2, p.179-85, 2003.

CORREAA, P.C.; CHRIST, D.; MARTINS, J.H.; MANTOVANI, B.H.M. Curvas de dessorção e calor latente de vaporização para as sementes de milho pipoca. Revista Brasileira de Engenharia Agricola e Ambiental, Campina Grande, v.2, n.1, p.75-9, 1998.

COSTA, N.A.; SANTOS, J.S.S.; SILVA, I.S. ELV Software. Campina Grande: Dep. Química/ UFCG, 1999. 105 p.

KALEEMULLAH, S.; KAILAPPAN, R. Latent heat of vaporization of moisture from red chillies. International Journal of Food Properties, Oxfordshire, v.8, n.2, p.199-205, 2005.

MATA, M.E.R.M.C.; DANTAS, L.A.; BRAGA, M.E.D. Programa computacional para simulação de secagem de grãos. Revista Brasileira de Produtos Agroindustriais, Campina Grande, v.1, n.1, p.33-50, 1999.

OLIVEIRA, J.R.; MATA, M.E.R.C.; DUARTE, M.E.M. Isotermas de dessorção de grãos de feijão macassar verde (Vigna unguiculata (L.) Walpers), variedade sempre-verde. Revista Brasileira de Produtos Agroindustriais, Campina Grande, v.6, n.1, p.61-70, 2004.

SHORE, H.; BRAUNER, N.; SHACHAM, M. Modeling Physical and thermodynamic properties via inverse normalizing transformation. Industrial \& Engineering Chemistry Research, Columbus, v.41, n.3, p.651-6, 2002.

SILVA, W.P.; MATA, M.E.R.M.C.; PRECKER, J.W.; SILVA, C.M.D.P.S.; SILVA, C.D.P.S.; SILVA, D.D.P.S.; LIMA, A.G.B. Uma equação empírica para determinação de teor de água de equilíbrio para grãos. Revista Brasileira de Produtos Agroindustriais, Campina Grande, v.7, n.1, p.71-82, 2005.

SILVA, W.P.; SILVA, C.M.D.P.S. Tratamento de dados experimentais. 2.ed. João Pessoa: UFPB Editora Universitária, 1998. 197 p. 
SILVA, W.P.; SILVA, C.M.D.P.S.; CAVALCANTI, C.G.B.; SILVA, D.P.S.; SOARES, I.B.; OLIVEIRA, J.A.S.O.; SILVA, C.D.P.S. LAB Fit Ajuste de Curvas: um software em português para tratamento de dados experimentais. Revista Brasileira de Ensino de Física, São Paulo, v.26, n.4, p.419-29, 2004.

SMITH, J.M.; VAN NESS, H.C.; ABBOT, M.M. Introdução à termodinâmica da engenharia química. Rio de Janeiro: LTC - Livros Técnicos e Científicos, 2000. 697 p.

TAYLOR, J. R. An introduction to error analysis. $2^{\text {nd }}$ ed. Sausalito, California: University Science Books, 1997. 327 p. 